\title{
Rotating perfect fluid models in general relativity
}

\section{Z. Perjés}

KFKI Research Institute for Nuclear and Particle Physics Budapest 114, P.O.Box 49, H-1525 Hungary

perjes@rmki.kfki.hu

Received 15 November 1999, accepted dd.mm.yyyy by ue

Abstract. The various schemes for studying rigidly rotating perfect fluids in general relativity are reviewed. General conclusions one may draw from these are: (i) There is a need to restrict the scope of the possible ansätze, and (ii) the angular behaviour is a valuable commodity. This latter observation follows from a large number of analytic models exhibiting a NUT-like behaviour. A method of getting around problem (ii) is presented on a simple example. To alleviate problem (i) for rigidly rotating perfect fluids, approximation schemes based on a series expansion in the angular velocity are suggested. A pioneering work, due to Hartle, explores the global properties of matched space-times to quadratic order in the angular velocity.

As a first example of the applications, it is shown that the rigidly rotating incompressible fluid cannot be Petrov type D.

Keywords: perfect fluids, general relativity

PACS: 04.40.Nr, 04.20.Jb

\section{Introduction}

In the past decades, numerous attempts have been made to construct analytic models of rotating perfect fluid bodies in general relativity. The static Schwarzschild metric of an incompressible fluid ball is known since 1916. The next development, Kerr's rotating solution for the vacuum domain, took no less than 46 years to happen. Three decades thereafter, the first, highly idealized model of a thin rotating disk of dust was found by Neugebauer and Meinel[1]. There are clear indications from this simple limit that the rotating relativistic star model is a task that is not easy to solve.

A perfect fluid medium in general relativity is characterized by a stress-energy tensor of the form

$$
T_{i k}=(\mu+p) \mathrm{u}_{i} \mathrm{u}_{k}-p g_{i k},
$$

where $p$ is the pressure, $\mu$ the density and $\mathrm{u}$ the four-velocity of the fluid, normalized by $\mathrm{u}_{i} \mathrm{u}^{i}=1$.

The field equations governing the fluid motion are Einstein's gravitational equations,

$$
R_{i k}-\frac{1}{2} g_{i k} R=k T_{i k}
$$


Barotropic fluids additionally obey an equation of state $p=p(\mu)$. The state is called stationary and axisymmetric when there exist two commuting Killing vectors,

$$
\xi_{t}=\frac{\partial}{\partial t} \quad \xi_{\varphi}=\frac{\partial}{\partial \varphi}
$$

such that $\xi_{t}$ is time-like and $\xi_{\varphi}$ space-like.

The fluid is said to be in circular rotation if the four-velocity $\mathrm{u}$ lies in the plane of the Killing vectors,

$$
\mathrm{u}=N\left(\xi_{t}+\Omega \xi_{\varphi}\right)
$$

Here $N$ is a normalization factor and $\Omega$ is the angular velocity of the fluid. Rigid rotation is characterized by the condition that $\Omega$ is a constant, and for differential rotation: $\mathrm{d} \Omega \neq 0$.

Spherical symmetry is characterized by a space-time filling family of 2-surfaces $d \vartheta^{2}+\sin ^{2} \vartheta d \varphi^{2} \quad$ (Delgaty and Lake[2], 1998).

The state is said to possess an equatorial symmetry when it is invariant under the substitution $\vartheta \rightarrow \pi-\vartheta$.

This paper presents a short review of the subject. The story begins in Sec. 2, with a Newman-Penrose presentation of the interior Schwarzschild metric. In Sec. 3, an overview is given of the various attempts to create more general models which, then, would describe rotation effects. It will be apparent hence, however, that success has yet eluded researchers. The main conclusion to be drawn from this situation is that, in the interest of better efficiency, there is a need for singling our the few ansätze that correctly encapsulate the physics of the rotating system. It is suggested here that a very efficient tool for doing this is given by the series expansion in powers of the angular velocity $\Omega$. This scheme has been worked out by Hartle 19 and used subsequently for a numerical study of stellar models 20, 21].

\section{The interior Schwarzschild metric}

The interior Schwarzschild metric, in its pristine form, is written

$$
d s^{2}=(A-\cos \chi)^{2} d t^{2}-R^{2}\left[d \chi^{2}+\sin ^{2} \chi\left(d \theta^{2}+\sin ^{2} \theta d \varphi^{2}\right)\right],
$$

where the radius $R$ of the sphere $S^{3}$ determines the density, $\mu=3 / R^{2}$ and the constant $A=3 \sqrt{1-r_{1}^{2} / R^{2}}$ encodes the radius $r_{1}$ of the star. The pressure of the fluid is

$$
p=\frac{1}{R^{2}} \frac{3 \cos \chi-A}{A-\cos \chi} .
$$

The familiar radial coordinate $r$ may be reintroduced by writing $\sin \chi=r / R$.

A Newman-Penrose analysis is conveniently carried out by use of a new, advanced, time coordinate $u$ such that

$$
d t=d u+\frac{R}{A-\cos \chi} d \chi
$$


Then the metric takes the form

$$
\begin{aligned}
d s^{2}= & (A-\cos \chi)^{2} d u^{2}+2 R(A-\cos \chi) d u d \chi \\
& -R^{2} \sin ^{2} \chi\left(d \theta^{2}+\sin ^{2} \theta d \varphi^{2}\right) .
\end{aligned}
$$

This is in a comoving coordinate system since we have

$$
\mathrm{u}=(A-\cos \chi)^{-1} \partial / \partial u .
$$

The null tetrad is chosen for the metric (8),

$$
\begin{aligned}
& \ell=\frac{\partial}{\partial \chi} \quad n=\frac{1}{R(A-\cos \chi)} \frac{\partial}{\partial u}-\frac{1}{2 R^{2}} \frac{\partial}{\partial \chi} \\
& m=\frac{1}{\sqrt{2} R \sin \chi}\left(\frac{\partial}{\partial \theta}+\frac{i}{\sin \theta} \frac{\partial}{\partial \varphi}\right) .
\end{aligned}
$$

Thus the four-velocity is given by the linear combination of the tetrad vectors

$$
\mathrm{u}=\frac{1}{2 R} \ell+R n .
$$

Inserting in the NP equations, we get the Ricci scalar

$$
\Lambda=\frac{1}{4 R^{2}} \frac{A-2 \cos \chi}{A-\cos \chi}
$$

and the Ricci-tensor components by

$$
\begin{aligned}
\Phi_{00} & =\frac{A}{A-\cos \chi} \quad \Phi_{11}=\frac{A}{4 R^{2}(A-\cos \chi)} \quad \Phi_{22}=\frac{1}{4 R^{4}} \frac{A}{A-\cos \chi} \\
\Phi_{01} & =\Phi_{02}=\Phi_{12}=0 .
\end{aligned}
$$

The spin coefficients satisfy the simple relations $\tau=\pi=\bar{\alpha}+\beta=\epsilon-\bar{\epsilon}=0$.

The Weyl spinor vanishes, $\Psi_{K}=0$, where $K=0,1, \ldots, 4$. The interior Schwarzschild solution is unique among the perfect fluid metrics in having the property of conformal flatness.

Collinson's theorem $([3], 1976)$ :

The only conformally flat axistationary perfect fluid is the interior Schwarzschild space - time .

\section{An (incomplete) compendium of the ansätze}

In this section we review the various schemes deployed for obtaining stationary and axisymmetric perfect fluid solutions of the gravitational equations. The entries are arranged in groups characterized by a common ansatz. For a detailed account of the state of the subject, $c f$. 㘬.

\section{- Petrov type}

Examples of type D solutions:

(i) Wahlquist 24, 1968 solution: This is a rigidly rotating perfect fluid with the equation of state $\mu+3 p=$ const. 
(ii) Senovilla [5], 1987 solution: The equation of state is $\mu-p=$ const. A later work by the same author, [6] 1992, presents a wide class of differentially rotating metrics containing a free function.

Relation to type $N$ vacuum:

Hoenselaers et al.[12], 1994 find a map from the type $\mathrm{N}$ vacuum field equations to the equations of rigidly rotating perfect fluids.

- Local Rotational Symmetry (LRS)

Stewart and Ellis 8], 1968 work out a classification scheme and find some analytic solutions. Marklund and Bradley, in a two-part paper [7], 1997 and 1999, employ the curvature representation in their study and obtain examples.

- Fluid kinematics

Chinea and Gonzalez-Romero[13, 1992 develop a formalism with differential forms adapted to the kinematics of the fluid.

Chinea 4 , 1993 uses this method for getting an analytic solution with differential rotation, by assuming that the acceleration of the fluid has a special form.

- Killing tensor

The vacuum Kerr metric possesses a nontrivial Killing tensor. This has instigated a research of perfect fluid states with a corresponding property by, among others, Kramer, Karlovini and Rosquist 15 and Goliath.

- Vanishing Simon tensor

Theorem (Kramer[14, 1985): for rigid rotation, the only possible equation of state is of the form $\mu+3 p=$ const.

Marklund et al.[9], 1997 generalize the notion of the Simon tensor for fluids in differential rotation.

- Electric-magnetic Weyl

Rotation is known to give rise to magnetic curvature. Since the interior Schwarzschild solution has zero conformal curvature, it has been surmised that the rotating interior Schwarzschild solution will have a purely magnetic curvature. Fodor et al.[22], 1999, develop a tetrad formalism in which the electric and magnetic parts of the curvature explicitly appear. Purely magnetic curvatures are discussed by Lozanowski and McIntosh [23].

- Lagrangian symmetry

Stephani [10], 1988 investigates the invariance properties of the perfect fluid Lagrangian. He finds that the system possesses far fewer symmetries than the vacuum Lagrangian.

- Static $\rightarrow$ stationary symmetry

Herlt 11], 1988 elaborates on methods by which to a given static state a rotating counterpart can be obtained.

- Geodesic eigenrays

Lukacs et al[16], 1983, obtain a fluid solution with geodesic eigenrays, exhibiting NUTlike behaviour.

From this impressive array of theoretical efforts, and from the fact that the only global solution properly matched to the ambient vacuum region is the infinitesimally thin, flat disk of dust of Neugebauer and Meinel[1], it transpires that the right ansatz has not yet been found.

Hence we may draw two important conclusions for this research:

CONCLUSION 1: There is a clear need to somehow restrict the scope of 
possible ansätze. The suggestion here is to resort to perturbation techniques. In the next section it will be shown that the approximation scheme developed originally by Hartle 19 is an efficient tool for selecting the physically acceptable schemes.

From the relatively large number of rotating fields with unacceptable causal behaviour, we draw

CONCLUSION 2: The angular behaviour is a valuable commodity.

\section{Linear perturbations}

In this section we demonstrate the advantages of a perturbative treatment in the slowrotation limit. Although previous works focussed mainly on numerical applications, we want to point out that perturbative methods can be used here like a torchlight when trying to find the causes of the difficulties met with analytic models.

(i) The lesson from the vacuum

Rotating vacuum fields are governed by the Ernst equation [17], (1968):

$$
(\xi \bar{\xi}-1) \Delta \xi=2 \bar{\xi} \nabla \xi \cdot \nabla \xi
$$

In spheroidal coordinates, this is written

$$
\begin{aligned}
(\xi \bar{\xi}-1)\left[\frac{\partial}{\partial x}\right. & \left.\left(x^{2}-1\right) \frac{\partial}{\partial x}-\frac{\partial}{\partial y}\left(1-y^{2}\right) \frac{\partial}{\partial y}\right] \xi \\
& =2 \bar{\xi}\left[\left(x^{2}-1\right)\left(\frac{\partial \xi}{\partial x}\right)^{2}-\left(1-y^{2}\right)\left(\frac{\partial \xi}{\partial y}\right)^{2}\right]
\end{aligned}
$$

Linearizing around the Schwarzschild solution $\xi=x$,

$$
\xi=x+i \xi_{1} \quad\left(\xi_{1} \text { real }\right)
$$

the derivative of the first-order function $\xi_{1}$ satisfies the Laplace equation:

$$
\Delta \frac{\partial^{2} \xi_{1}}{\partial x^{2}}=0
$$

Hence

$$
\frac{\partial^{2} \xi_{1}}{\partial x^{2}}=\sum_{l=2}^{\infty} a_{l} Q_{l}(x) P_{l}(y)
$$

where $P_{l}(y)$ is the Legendre polynomial of order $l$ and

$$
\xi_{1}=\sum_{l=2}^{\infty} a_{l} \int\left(\int Q_{l}(x) d x\right) d x P_{l}(y)+a y .
$$


The analytic solutions of the Ernst equation found by Tomimatsu and Sato (18], 1972) have the rational form

$$
\xi=\alpha / \beta .
$$

Fortuitously, the integral (19) is of polynomial character. The polynomials in $\xi_{1}$ have been used for the leading terms in $\alpha$.

\section{(ii) Perfect fluids}

Hartle [19] (1967) casts the metric of a slowly and rigidly rotating perfect fluid in the following form:

$$
d s^{2}=e^{\nu} d t^{2}-e^{\lambda} d r^{2}-r^{2}\left[d \vartheta^{2}+\sin ^{2} \vartheta(d \varphi-\omega d t)^{2}\right]+\mathcal{O}\left(\Omega^{2}\right) .
$$

From the large- $r$ behaviour it follows that the rotational potential $\omega$ is a function of the radius alone, $\omega=\omega(r)$.

From the Schwarzschild solution (5) for an incompressible fluid we get:

$$
e^{\nu}=(A-\cos \chi)^{2}, \quad e^{\lambda}=\frac{R^{2}}{\sin ^{2} \chi} .
$$

The potential $\omega$ satisfies Heun's equation

$$
(A-z)\left(z^{2}-1\right) \frac{d^{2} \omega}{d z^{2}}-\left(3 z^{2}-5 A z+2\right) \frac{d \omega}{d z}+4 A \omega=0
$$

with $z=\cos \chi$.

This equation has proved to be a stumbling block in the way of progress for three decades. Naïve attempts at removal do fail. For example, one may ask for the effect of coordinate change. Since the $\omega$ equation is a special case of the six-parameter Heun Eq., it would seem conceivable that in a better coordinate system, solution is more amenable. Such a new coordinate system is provided, for example, by the property of the interior Schwarzschild metric that it is conformally flat. As a consequence, the metric can be conformally mapped to the Einstein Universe (as the case is with the Minkowski spacetime):

$$
d s^{2}=\Omega^{2}\left[d t^{2}-d x^{2}-\sin ^{2} x\left(d \vartheta^{2}+\sin ^{2} \vartheta d \varphi^{2}\right)\right] .
$$

The conformal factor is $\Omega^{2}=R^{2}\left(A^{2}-1\right) /(A-\cos x)^{2}$. The transformation to the conformal form 24) is as follows, $\sin x=\left(A^{2}-1\right)^{1 / 2}(A-\cos \chi)^{-1} \sin \chi$. We find, however, that the perturbation equation is of the Heun type again.

In a like manner, attempts to change the dependent variable have failed to turn up a solvable equation. A straightforward thought here is to try and use the Ernst potential in place of the rotation potential $\omega$ in the metric

$$
\begin{aligned}
d s^{2}= & f(d t+\omega d \varphi)^{2} \\
& -f^{-1}\left[e^{2 \gamma} d r^{2}+\left(1-r^{2}\right)\left(\left(1-y^{2}\right)^{-1} d y^{2}+\left(1-y^{2}\right) d \varphi^{2}\right)\right],
\end{aligned}
$$


where $y=\cos \vartheta$. The field equation for the Ernst potential $\xi$ is

$$
(\xi \bar{\xi}-1) \Delta \xi-2 \bar{\xi} \nabla \xi \cdot \nabla \xi=\frac{1}{2} k(\xi \bar{\xi}-1)(\xi+1)^{2}(\mu+3 p)
$$

with $p$ the pressure and $\mu$ the density of the fluid.

Integration of the Bianchi identity yields

$$
(\mu+p) f^{1 / 2}=\text { const. }
$$

For the interior Schwarzschild metric, $\gamma=0$, and

$$
f=(A-r)^{2}, \quad k \mu=3, \quad k p=\frac{3 r-A}{A-r} .
$$

Let the rotational perturbation $\xi_{1}$ be introduced,

$$
\xi=\frac{1+f}{1-f}+i \xi_{1} .
$$

The linearized field equation for $\xi_{1}$ is separable; the $y$ dependence satisfies the Legendre equation of index $l$.

It is possible to integrate the radial equation for $l=0$ :

$$
\xi_{1}=\frac{1}{\left[(r-A)^{2}-1\right]^{2}}\left\{C_{1}-C_{2}\left[\frac{r^{2}+3 A r+A^{3} r-2-3 A^{2}}{\sqrt{r^{2}-1}}+3 A \ln \left(r+\sqrt{r^{2}-1}\right)\right]\right\} .
$$

This would appear quite encouraging. However, from asymptotic matching, one finds that $\xi_{1}$ is proportional to $y$, and hence we need the integral for $l=1$, which is not known in a closed form.

\section{Two theorems}

In this section we describe how to restrict the scope of choice in order to meet Conclusion 1 , by applying the perturbative treatment to various systems.

\subsection{Theorem on incompressible fluids}

To second order in $\Omega$, the metric of the rigidly rotationg incompressible fluid is 19

$$
\begin{aligned}
d s^{2}= & (1+2 h)(A-\cos \chi)^{2} d t^{2}-R^{2}(1+2 k) \sin ^{2} \chi \sin ^{2} \vartheta(d \varphi-\omega d t)^{2} \\
& -R^{2}(1+2 m) d \chi^{2}-R^{2}(1+2 n) \sin ^{2} \chi d \vartheta^{2} .
\end{aligned}
$$

Here $\omega$ is of first order, and $h, k, m, n$ of second order in the angular velocity $\Omega$.

A comoving tetrad may be chosen

$$
\begin{aligned}
& e_{0}^{t}=\frac{1-h}{A-\cos \chi}+(\omega-\Omega)^{2} \frac{R^{2} \sin ^{2} \vartheta \sin ^{2} \chi}{2(A-\cos \chi)^{3}} \\
& e_{0}^{\varphi}=\frac{\Omega}{A-\cos \chi}, \quad e_{1}^{\chi}=\frac{1-m}{R} \\
& e_{2}^{\vartheta}=\frac{1-n}{R \sin \chi}, \quad e_{3}^{t}=\frac{R \sin \vartheta \sin \chi(\omega-\Omega)}{(A-\cos \chi)^{2}} \\
& e_{3}^{\varphi}=\frac{k-1}{R \sin \vartheta \sin \chi}+\left(\omega^{2}-\Omega^{2}\right) \frac{R \sin \vartheta \sin \chi}{2(A-\cos \chi)^{2}} .
\end{aligned}
$$


The vorticity is given by the first-order rotation coefficients

$$
\begin{aligned}
& \omega_{1}=\frac{1}{2(A-\cos \chi)}\left[2 \cos \vartheta(\Omega-\omega)-\sin \vartheta \frac{\partial \omega}{\partial \vartheta}\right] \\
& \omega_{2}=\frac{\sin \vartheta}{2(A-\cos \chi)^{2}}\left[2(\omega-\Omega)(A \cos \chi-1)+\sin \chi(A-\cos \chi) \frac{\partial \omega}{\partial \chi}\right] .
\end{aligned}
$$

The changes in the other rotation coefficients are of the second order.

The magnetic part $\mathrm{H}_{i}$ of the Weyl tensor is of order $\Omega$,

$$
\begin{aligned}
& H_{1}=\frac{\cos \vartheta}{R(\cos \chi-A)} \frac{d \omega}{d \chi}, \quad H_{2}=-\frac{1}{2} H_{1} \\
& H_{3}=-\frac{\omega_{2} \cos \chi}{R \sin \chi}+\frac{\sin \vartheta}{R \sin \chi} \frac{\omega-\Omega}{A-\cos \chi} .
\end{aligned}
$$

The electric part $E_{i}$ is of order $\Omega^{2}$. The condition for the space-time to be Petrov type II, to quadratic order, is $H_{3}=0$ and $H_{1}=H_{2}$. Hence the Petrov type of this incompressible fluid cannot be II.

The Petrov type is D if $H_{3}^{2}-2 H_{1}^{2}-2 H_{2}^{2}-5 H_{1} H_{2}=0$. The general solution of this condition has the form

$$
\omega=C(A / \cos \chi-1)^{2} .
$$

This is not a solution of the Heun equation (23) which proves the following

THEOREM[26]: A rigidly rotating incompressible fluid ball in an asymptotically flat vacuum exterior cannot be Petrov type D.

\subsection{Theorem on the Wahlquist solution}

The Wahlquist metric is an example of type $\mathrm{D}$ solutions. It is given by 24

$$
\begin{aligned}
d s^{2}=f(d t-A d \varphi)^{2} & -r_{0}^{2}\left(\zeta^{2}+\xi^{2}\right)\left[\frac{d \zeta^{2}}{\left(1-\mathrm{k}^{2} \zeta^{2}\right) h_{1}}\right. \\
& \left.+\frac{d \xi^{2}}{\left(1+\mathrm{k}^{2} \xi^{2}\right) h_{2}}+\frac{h_{1} h_{2}}{h_{1}-h_{2}} d \varphi^{2}\right],
\end{aligned}
$$

where

$$
\begin{aligned}
f & =\frac{h_{1}-h_{2}}{\zeta^{2}+\xi^{2}} \quad A=r_{0}\left(\frac{\xi^{2} h_{1}+\zeta^{2} h_{2}}{h_{1}-h_{2}}-\xi_{A}^{2}\right) \\
h_{1}(\zeta) & =1+\zeta^{2}+\frac{\zeta}{\kappa^{2}}\left[\zeta-\frac{1}{\mathrm{k}} \sqrt{1-\mathrm{k}^{2} \zeta^{2}} \arcsin (\mathrm{k} \zeta)\right] \\
h_{2}(\xi) & =1-\xi^{2}-\frac{\xi}{\kappa^{2}}\left[\xi-\frac{1}{\mathrm{k}} \sqrt{1+\mathrm{k}^{2} \xi^{2}} \operatorname{arcsinh}(\mathrm{k} \xi)\right] .
\end{aligned}
$$

The constant $\xi_{A}$ is defined by $h_{2}\left(\xi_{A}\right)=0$.

The pressure and density are

$$
p=\frac{1}{2} \mu_{0}\left(1-\kappa^{2} f\right) \quad \mu=\frac{1}{2} \mu_{0}\left(3 \kappa^{2} f-1\right) .
$$


The equation of state is

$$
\mu_{0}=\mu+3 p=\frac{2 \mathrm{k}^{2}}{\kappa^{2} r_{0}^{2}} .
$$

THEOREM [27]: By quadratic approximation in $\Omega$,

The Wahlquist metric cannot be matched to an asymptotically flat vacuum exterior.

\section{$6 \quad$ How to secure the angular behaviour?}

I do not really have a satisfactory answer to Conclusion 2 concerning the angular behaviour. All I can offer here is a particular recipe by which one creates a relativistically rotating version of a certain static system. There is no guarantee, however, that the space-time so obtained will have the desired type of matter source. Succinctly stated, the idea is as follows. The constant-time sections of the Schwarzschild fluid ball (5) are $S^{3}$ spheres. Now an $S^{3}$ may be squeezed and simultaneously set in rotation about the $z$ axis following these steps:

- Embed $\mathcal{S}^{3}$ in $E^{4}$ with the metric

$$
d s^{2}=\left(d x^{1}\right)^{2}+\left(d x^{2}\right)^{2}+\left(d x^{3}\right)^{2}+\left(d x^{4}\right)^{2} .
$$

Introduce polar coordinates $(R, \chi, \vartheta, \varphi)$,

$$
\begin{array}{r}
x^{1}+i x^{2}=R \sin \chi \sin \vartheta e^{i \varphi} \\
x^{3}=R \sin \chi \cos \vartheta \\
x^{4}=R \cos \chi .
\end{array}
$$

The surface $\mathcal{S}^{3}$ is given by $R=$ const.

- Squeeze $\mathcal{S}^{3}$ and set simultaneously in rotation by putting

$$
\chi \rightarrow \chi+i a
$$

in $x^{1}+i x^{2}$. Thus the $R=$ const. surface is flattened, and has the metric

$$
\begin{aligned}
d s^{2} & =\left(\sin ^{2} \chi+\sinh ^{2} a \cos ^{2} \vartheta\right)\left(d \vartheta^{2}+\sin ^{2} \vartheta d \varphi^{2}\right) \\
& +\sinh a \cosh a \sin ^{2} d \chi d \varphi-\sinh ^{2} a \sin ^{4} \vartheta d \varphi^{2} .
\end{aligned}
$$

- Embed $\mathcal{S}^{3}$ in the Einstein Universe. For $R=1$, the metric is of the form

$$
d s^{2}=d t^{2}-d \chi^{2}-\sin ^{2} \chi\left(d \vartheta^{2}+\sin ^{2} \vartheta d \varphi^{2}\right) .
$$

- Soak up the slack $d \chi d \varphi$ term by introducing the advanced time coordinate

$$
u=t+\chi \text {. }
$$

The space-time metric then takes the form:

$$
\begin{aligned}
d s^{2} & =\left(d u-\sinh a \sin ^{2} \vartheta d \varphi\right)^{2} \\
- & 2\left(d u-\sinh a \sin ^{2} \vartheta d \varphi\right)\left(\cosh a d \chi-\sinh a \sin ^{2} \vartheta d \varphi\right) \\
- & \left(\sin ^{2} \chi+\sinh ^{2} a \cos ^{2} \vartheta\right)\left(d \vartheta^{2}+\sin ^{2} \vartheta d \varphi^{2}\right) .
\end{aligned}
$$


This metric represents a squeezed and rotated Einstein universe. In the limit as $R \rightarrow \infty$, we recover Minkowski space-time.

CONJECTURE : By continuity in the rotation parameter $a$,

There is an open neighbourhood of Minkowski space-time in the minisuperspace of squeezed Einstein universes that is causally well-behaved.

Application of this procedure to the interior Schwarzschild metric yields rotating models with various stress-energy tensors, depending on details of the embedding.

It is a pleasure to thank the Organizers of the Journees Relativistes Conference for providing a seamless platform for scientific activity and for hospitality. Part of this work has been done under the contract OTKA T022533.

\section{References}

[1] G.Neugebauer and R.Meinel, Astrophys. J. 414, (1993) L97

[2] M.S.R.Delgaty and K. Lake, Comput. Phys. Commun. 115 (1998) 395, gr-qc/9809013

[3] C.D.Collinson, Gen.Rel.Grav. 7, (1976) 419

[4] F.J.Chinea and L.M.González-Romero (Eds.): Rotating Objects and Relativistic Physics, Proceedings, El Escorial, Lecture Notes in Physics Vol. 423, Springer, 1993

[5] J.M.M.Senovilla, Class. Quantum Grav. 4, (1987) L115

[6] J.M.M.Senovilla, Class. Quantum Grav. 9, (1992) L167

[7] M.Marklund, Class. Quantum Grav. 14, (1997) 1267, M.Marklund and M.Bradley, Class. Quantum Grav. 16, (1999) 1577

[8] J.M.Stewart and G.F.R.Ellis, J. Math. Phys. 9, (1968) 1072

[9] M.Marklund and Z.Perjés, J. Math. Phys. 38, (1997) 5280

[10] H.Stephani, J. Math. Phys. 29, (1988) 1650

[11] E.Herlt, Gen. Rel. Grav. 20, (1988) 635

[12] C.Hoenselaers, R.Ferwagner and W.Schief, in Relativity Today, Edited by R.P.Kerr and Z.Perjés, Akadémiai Kiadó, Budapest, 1994, p. 47

[13] F.J.Chinea and L.M.González-Romero, Class. Quantum Grav. 9, (1992) 1271

[14] D.Kramer, Class. Quantum Grav. 2, (1985) L135

[15] M.Karlovini and K.Rosquist, Gen.Rel.Grav. 31, (1999) 1271, gr-qc/9807051

[16] B.Lukacs et al., Gen. Rel. Grav. 15, (1983) 567

[17] F. J. Ernst, Phys. Rev. 167, (1968) 1175

[18] A. Tomimatsu and H. Sato, Phys. Rev. Letters 29, (1972) 1344

[19] J.Hartle, Astrophys. J. 150, (1967) 1005

[20] J.B.Hartle and K.S.Thorne, Astrophys. J. 153, (1968) 807

[21] S.Chandrasekhar and J.C.Miller, Mon.Not.R.Astr.Soc. 167, (1974) 63

[22] G.Fodor, M.Marklund and Z.Perjés, Class. Quantum Grav. 16, (1999) 453

[23] C.Lozanowski and C.B.G.McIntosh, Gen. Rel. Grav. 31, (1999) 1355

[24] H. D. Wahlquist, Phys. Rev. 172, (1968) 1291

[25] J. M. Whittaker, Proc. Roy. Soc. A 306, (1968) 1

[26] G.Fodor and Z.Perjés, Petrov types of slowly rotating fluid balls, gr-qc/9911068

[27] M.Bradley, G.Fodor, M.Marklund and Z.Perjés, The Wahlquist metric cannot describe an isolated rotating body, gr-qc/9910001, to appear in Class. Quantum Grav. 